\title{
Intelligent device for diagnosis of dam's condition
}

\author{
F. V. Savrasov ${ }^{1}$, A. A. Shamin ${ }^{1}$, E. A. Pershin ${ }^{1}$, R. V. Meyta ${ }^{1}$, A. N. Moiseev ${ }^{2}$ \\ ${ }^{1}$ Institute of Cybernetics \\ National Research Tomsk Polytechnic University \\ Tomsk, Russia \\ savrasov@tpu.ru \\ ${ }^{2}$ Tomsk State University \\ Tomsk, Russia \\ moiseev.tsu@gmail.com
}

\begin{abstract}
This paper describes the purpose of device for data collection and transmission to diagnose the condition of the dam. The structure of hardware and software components of the device called "Varyag" is depicted.
\end{abstract}

Keywords - dam; controller; sensor; GPRS; Modbus; JSON.

\section{INTRODUCTION}

The intelligent device called "Varyag" was designed to monitor of hazardous situations that arise at result of dam destructions or a natural disasters.

Dam is a hydraulic engineering work that partitions off a watercourse or body of water by a septum for lifting the water level. Also it serves to concentrate the pressure at the location of facilities and the creation of the reservoir. The collapse of the dam may lead the events such as the flooding of settlements, the destruction of structures by pressure of water, flooding of agricultural enterprises. Any of these events entails huge economic cost of restoring buildings and rescue operations. As a consequence, it is necessary to monitor the condition of the dam, and, in the event of critical values of the water level, to alert the dam's control center, which sends people to check and fix problems [1].

The system for diagnostic of dam's state consists of several basic units:

- data collection center, where specialists are attented who needs to respond to critical situations (eg MES);

- stand-alone device (controller) that is installed on the dam;

- sensors for monitoring of the dam's condition.

Each data collection center may collect information from multiple controllers that are installed on the same or different dams. An example of this system is shown in Fig. 1.

Often there are problems with power supply networks. This networks are not exists in areas, where located controller is located. As result, the controllers must have stand-alone mode with a battery-powered system. Each controller has a set of sensors to analyze conditions of the dam (including the measurement of the water level). The measured data transmits to the center at specified intervals.

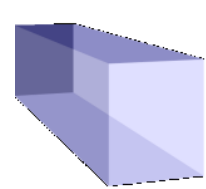

Dam 1

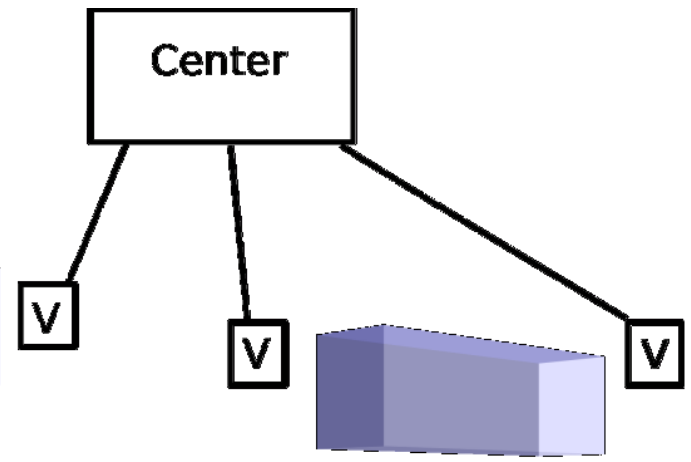

Dam 2
Fig. 1. Example of dam's diagnostic system

In the process of developing the device some basic conditions for the functioning of the hardware and software of the controller were determined:

- supporting the required number of sensors, with the possibility of adding new sensors;

- diagnostics of sensors for their refusal (the device must notify operators about fault sensors);

- sending warning messages to operators about the discharge of the battery and automatic device shot-down at the critical discharge of the battery;

- redundant the communication channels;

- supporting of VPN-network (this is necessary to protect the device from unauthorized access); for this, all data transfer operations need to be performed inside the local network.

\section{STRUCTURE OF THE DEVICE}

Controller "Varyag" (see Fig. 2) is designed to work in extreme conditions. It is performed in a uniform plastic case with a level of protection against external influences - IP30, providing installation of the device on the mounting rail (type 
DIN 35). Operating temperature range of the controller - from minus 40 to plus $60{ }^{\circ} \mathrm{C}$ with relative humidity up to $95 \%$. The controller is resistant to high frequency vibration and shock, also seismically resistant to 9 points earthquakes on the MSK64 [2].

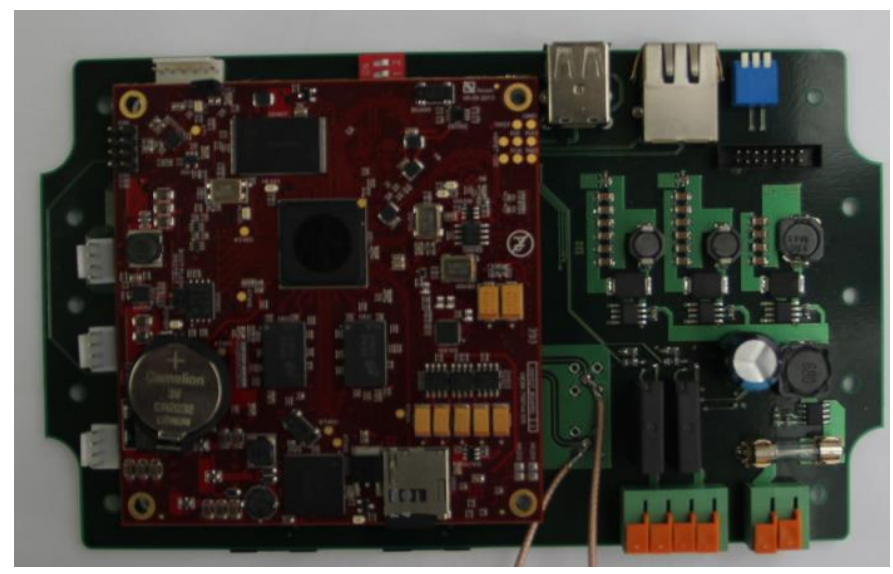

Fig. 2. Controller "Varyag" without box

Controller "Varyag" consists of several key components. Block diagram of the controller is shown in Fig. 3.

CPU board MEVK.758744.006 performed with a processor core of Cortex-A8 with installed Linux operating system and executable programs. Peripheral controller provides the interface CPU board MEVK.758744.006 with external devices connected to the interface RS485/RS422. Peripheral controller communicates with the CPU using ModBus protocol and has a fixed address " 247 ".

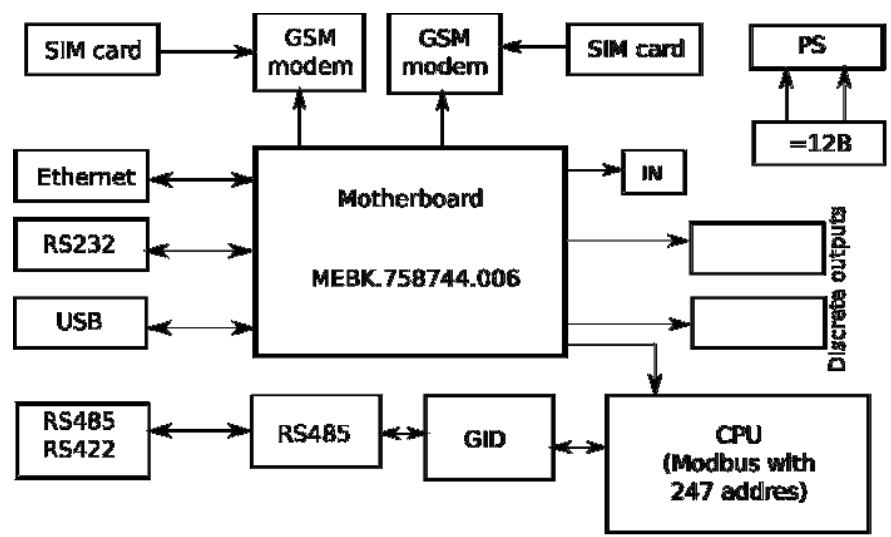

Fig. 3. Block diagram of "Varyag"

Indication note (IN) of the controller includes an indicator, located on the top cover of controller. Indicator lights when the unit is powered up. Power supply (PS) converts the voltage controller (from 10 to $18 \mathrm{~V}$ ) in the voltage required for the job. Galvanic isolation device (GID) is made on the optocouplers and provides galvanic isolation circuit interface RS485/RS422 from the input circuits and power controller.

The battery unit provides autonomous operation of the controller during the week. Nominal voltage range DC power varies from 10 to $18 \mathrm{~V}$. Power consumption is less than 5 watts, it shows the efficiency of the developed device. Monitoring of battery status is provided by the controller managing the peripheral interfaces.

\section{SENSORS}

There are multiple types of sensors, which can be connected to device: distance measurement module with a built-in depth gauge (MB-110), and a module that defines discrete signals (DI-100) Fig. 4

Typical arrangement of the sensors on the dam is shown in

The figure shows: $A$ - the dam; $B$ - the current water level; $C$ - discrete sensors DI-100; $D$ - sensor MB-110.

MB-110 module is used in a device for determining the distance from the critical water level to the current water level. Each module is equipped with two sensors (depth gauge) for the location at two different points. To determine the distance to the water a special ultrasonic transducer which can measure the distance with an accuracy of $2 \mathrm{~cm}$ is used. The action of the sensor extends over a distance of $25 \mathrm{~cm}$ to 6 meters [3]

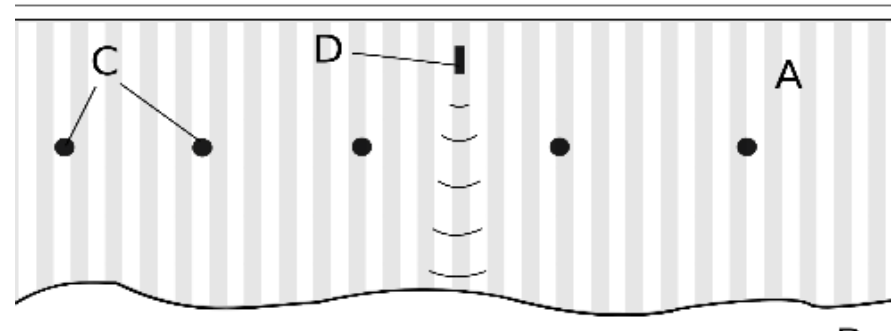

B

Fig. 4. Example of the sensor locations on the dam

DI-100 module has been constructed specifically for the controller "Varyag". The module is designed to input the four discrete signals and transmitting information via RS-485. This module is used to determine the level of water at the sensor's point of installing. Physically, the sensor detects, in dry or wet environment is a measuring device. The module is equipped with four discrete sensors. Each sensor is installed at a critical level of water rise. Upon reaching water the sensor's level, the system determines that the critical water level exceeded and sends a corresponding signal to the center.

Each measurement unit is placed in a plastic box and has a protective characteristics as the main controller, allowing their use under extreme conditions. The motherboard also has support for two internal discrete sensors that are used for service purposes, such as determining the opening of the container in which the controller is placed. When opening the container, the controller informs the center about this event. That allows to determine unauthorized access to the device.

\section{COMMUNICATION CHANNELS AND DATA TRANSFER PROTOCOLS}

The device has a support for multiple types of communication channels. The controller has interfaces such as 
RS232, RS485, USB, Ethernet, GPRS. The communication channels for communication with the center is used only Ethernet and GPRS channels. The board has one Ethernet 10/100 port and two GSM/GPRS modem. Each GPRS transceiver supports two SIM cards in the DSSS-mode. Using the Ethernet port, it is possible to create up to eight channels of communication online. In sum, it is possible to create 12 channels for data transfer between the center and the device: 8 Ethernet channels and 4 GPRS channel.

For data transfer inside the controller, the protocol Modbus [4] is used. In the developed device, the communication is organized between all the components of the controller (battery, GPRS-modem) and connected devices (modules MB-110 and DI-100) via Modbus. All internal components on the board have one fixed address Modbus, equal to 247. Each plug-in sensor has its own address from one to 246. Built-in sensors located on the motherboard controller connect with the central processor also via ModBus.

For physical control of certain components, the controller has two relays that can be used to connect and disconnect some of the components with power supply. At present moment, one relay is used to disconnect all the sensors at a period when no measurements are made. This has a positive effect on the energy consumption of the developed device.

Packets to transfer data between the center and the device encapsulate with format JSON (a text-based data exchange format based on JavaScript) [5]. Example of JSON-row is shown in Fig. 5.

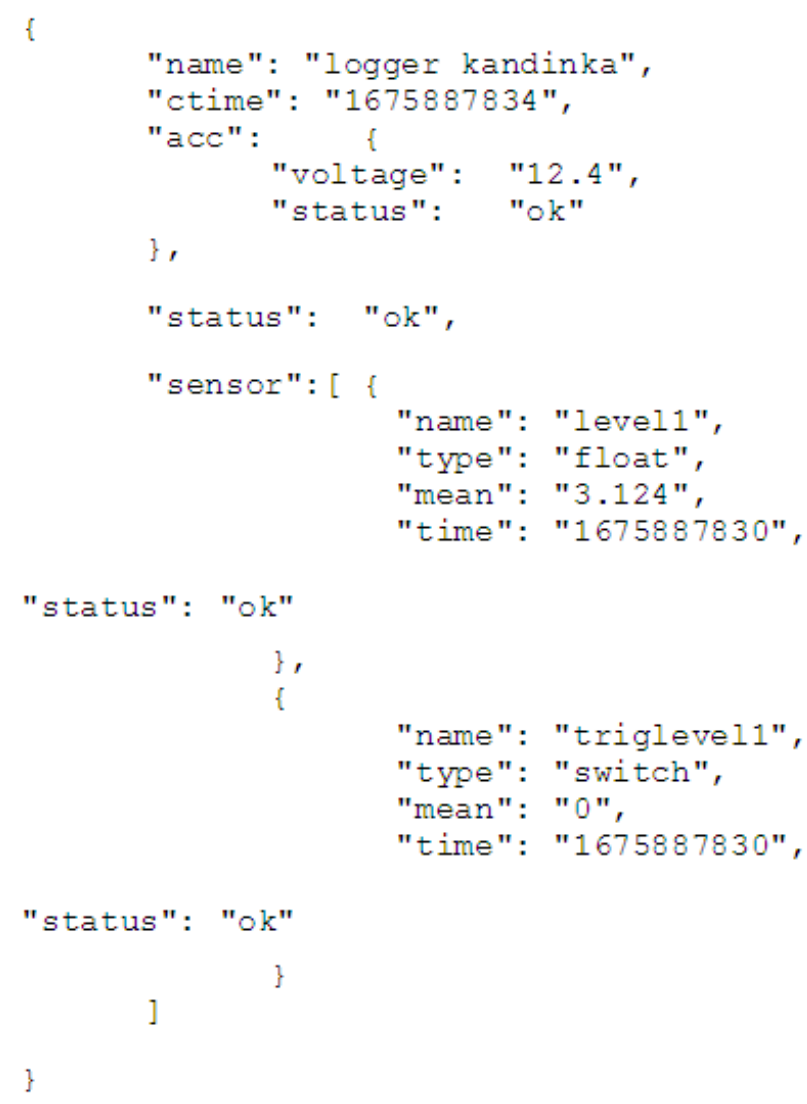

Fig. 5. Example of a data packet containing the current sensor readings
JSON can be compared with XML format, but it is more compact. This format was chosen because it has a number of advantages:

- simplicity;

- compactness (packet size is less than analogues such as XML);

- readability;

- flexibility (the ability to transmit data of various types, as well as other objects and arrays);

- the possibility of using the data exchange between different programming languages and operating systems.

The last position is particularly important because the controller has operating system Linux, and the data center has Windows-based OS. The software of the controller and data center is written in different programming languages. The possibility of compact package for data transmission at the center due the slow speed of communication channels also is important. This circumstance requires when GPRS-modem is using (it works in extreme environments because there is no possibility of establishing Ethernet connections in such conditions).

The disadvantage of JSON format is the lack of ability to send a link to another JSON-object, but within this work it is not essential.

Formed JSON package contains the following information:

- timestamp (when the package was created);

- the device name (code);

- information about the status of the battery (battery condition and charge level);

- device status (running or disabled).

During the transmitting of the measured data, an array of objects that describe the state of the evidence and sensors is added.

To reduce the amount of data transfer over the network, packets are compressed using the library "zlib", and then sent to the center. Messages received from the center also have the JSON format and contained in archives. The length of the message is less than one kilobyte; that reduces the chances of breaking the connection during the transmission of messages. In case of an error when transmitting a message, or lack of communication channels, packets are placed in the database and sent to the center after connection's repair.

\section{COMPARISON WITH ANALOGUES}

It had been noted during the process of device's development that amount of instruments, which are absolutely similar to "Varyag", is very little. The projected device can be compared to the system of data collection CR800, developed by Campbell Scientific. The main features of CR800 are:

- 9 analog sensors; 
- battery voltage from 9,6 to $16 \mathrm{~V}$;

- the support of PakBus, Modbus, and DNP3;

- interfaces channels such as satellite, Ethernet, Radio, GPRS, RS232, RS485;

- built-in display.

The developed controller has an advantage over CR800 in the number of supported sensors (246) and battery capacity, but it supports less data transfer protocols and has not built-in display (it is option). Also in the controller "Varyag" an adaptive algorithm for polling the sensors and formation of messages is used (in similar devices this function is not supported, they use only predefined periods of polling).

This algorithm has been designed to save energy consumption of the device. It is based on two assumptions:

- the largest amount of energy is consumed in the survey of sensors and data transmission over communication channels;

- the rate of changing the water level is not constant in time (during another events, such as rainfall, damages of the dam et al., the water level may vary considerably faster than in normal conditions).

Adaptive algorithm of polling is described below.

1. Reading the next result of sensor's measurement of the water level.

2. Calculation of next polling time $t_{1}$.

3. Creating and sending messages.

4. Waiting for the moment of time $t_{1}$.

5. Go to step 1 .

For further calculations, the difference between values of water level in the current and previous survey is used.

Denote by $P_{0}$ the polling period in the normal condition; $D_{a}$ - is the value of water level's change, at which frequency of polling begins to increase; $D_{c u r}$ - is the difference of water level's value between the current and previous measurement; $P_{1}$ - is the period of polling after the recalculation; $t_{1}-$ the time of the previous polling. In case of $D_{\text {cur }}$ is less or equals to $D_{a}$, the value of $P_{1}$ is set to $P_{0}$. The formula of evaluating $P_{1}$ in case of $D_{\text {cur }}$ is more then $D_{a}$ is

$$
P_{1}=\left(\left(P_{1}\right)^{2} \cdot D_{a}\right) /\left(P_{0} \cdot D_{\text {cur }}\right)
$$

and the formula for calculating the time of the next polling is

$$
t_{1}=P_{1}+t_{0}
$$

This algorithm uses predefined values of maximum and minimum periods of formation data from the sensors. If, as a result of algorithm's operation, calculated period exceeds these values, the system generates data at a given minimum or maximum periods of time ( $P_{1}$ sets to the according value), but it occurs after period's calculation in accordance with (1).

The using an adaptive algorithm can improve device's efficiency and reduce the amount of data transmitted over the communication channels. If the changing of water level is in the normal range, then the polling occurs over a period specified by the center (it saves battery charge).

\section{CONCLUSION}

In comparison with devices of similar purpose, the controller "Varyag" has both advantages and disadvantages. The advantages of this system include:

- simplicity (a small number of components is used, the device comes in a plastic container);

- price (relatively to similar devices, the cost of the system is lower);

- readability;

- dimensions (the device is compact and can be placed in a limited space);

- adaptive algorithm for polling the sensors.

The disadvantages of the controller are the limitations of the hardware and software system (it is intended to measure the water level and alert, and thereby an advantage in price and dimensions follows)).

\section{ACKNOWLEDGMENT}

This work has been supported as a part of the state task "Nauka".

\section{REFERENCES}

[1] V.P. Singh, R.N. Yadava, Water Resources System Operation: Proceedings of the International Conference on Water and Environment. Bhopal, India: Allied Publishers, 2003.

[2] S. Chattopadhyay, Embedded System Design. Delhi, India: PHI Learning Pvt. Ltd., 2013.

[3] J. Fraden, Handbook of Modern Sensors: Physics, Designs, and Applications. New York, USA: Springer, 2010.

[4] P. Chipkin, Modbus For Field Technicians. Vancouver, CA: Chipkin Automation Systems, 2011.

[5] S.S. Sriparasa, JavaScript and JSON Essentials. Packt Publishing, 2013. 